\title{
Numerical Simulation of Molten Metal Droplet Behavior in Gas Metal Arc Welding by Three-Dimensional Incompressible Smoothed Particle Hydrodynamics Method*
}

\author{
Hisaya Komen\#, Masaya Shigeta, Manabu Tanaka \\ Joining and Welding Research Institute, Osaka University, Osaka, Japan \\ Email: "h.komen@jwri.osaka-u.ac.jp
}

How to cite this paper: Komen, H., Shigeta, M. and Tanaka, M. (2018) Numerical Simulation of Molten Metal Droplet Behavior in Gas Metal Arc Welding by Three-Dimensional Incompressible Smoothed Particle Hydrodynamics Method. Journal of Flow Control, Measurement \& Visualization, 6, 66-81.

https://doi.org/10.4236/jfcmv.2018.62007

Received: December 4, 2017

Accepted: February 22, 2018

Published: April 12, 2018

Copyright $\odot 2018$ by authors and Scientific Research Publishing Inc. This work is licensed under the Creative Commons Attribution International License (CC BY 4.0).

http://creativecommons.org/licenses/by/4.0/

\begin{abstract}
The numerical model was developed using a SPH (Smoothed Particle Hydrodynamics) method and the projected transfer phenomena during a GMA (Gas Metal Arc) welding were simulated by the model to clarify mechanisms of the phenomena. As a result, the droplet transfer mode obtained from this calculation was regarded as a projected transfer mode in which the liquid column grew about $1 \mathrm{~mm}$ and a droplet grew up until its diameter became large the same as a wire diameter, after that it was detached from the tip of the column. In addition, 10 droplets were formed for $0.1 \mathrm{~s}$ through these growth and detachment processes at the tip of a wire. To compare with the numerical results, actual GMA welding was carried out and molten metal droplet transfers were taken by high speed camera. The diameter of a wire, the length of a liquid column, the velocity of a droplet right before it reached a weld pool obtained by simulation showed good agreement with experiment.
\end{abstract}

\section{Keywords}

SPH Method, Gas Metal Arc Welding, Molten Metal Droplet Transfer, Simulation

\section{Introduction}

GMA (Gas Metal Arc) welding is one of the joining processes in which the welding is conducted with heating and melting of a wire electrode. This process is widely used in the industry because it is easy to automatize and apply to vari-

${ }^{\star}$ A part of this work was presented at 14th International Conference Fluid Dynamics (ICFD14) in Sendai, 1-3 Nov. 2017. 
ous materials. In GMA welding process, a wire is heated and melted by mainly the Joule heating and the electron inflow. Then, a molten metal droplet is formed at the tip of a wire. The droplet grows with time evaluation and it is detached by influences of the gravity and the electromagnetic force. Then, it is transported to a weld pool by the shielding gas and the gravity. These behaviors are called molten metal droplet transfer phenomena and it is one of the important factors to determine the amount of spatters during a welding. These phenomena are changed from the globular transfer mode to the spray transfer mode with increase in a welding current [1]. Projected transfer mode is one of the spray transfer modes. In this mode, a droplet is detached near the solid-liquid interface and the detachment periodically occurs. So it is easy to control the droplet behavior in the projected transfer mode and this mode is suitable for a GMA welding. However in a GMA welding, the penetration shape is affected by the droplet transfer [2]. It is one of the important factors to determine the welding quality. Therefore, understanding and control of the droplet transfer phenomena are necessary to improve the quality of a weld part. Recently many researches using numerical simulation have been conducted to understand the droplet transfer phenomena in this GMA welding. For example, Ogino et al. simulated the arc phenomena and the droplet transfer phenomena during pulse GMA welding in two-dimensional axisymmetric domain [3]. Using trapezoidal current waveform which had the difference about 400 A between a base current and a peak current, they showed the number of transported droplets per one pulse changed by the peak current time. Then, it was simulated that the droplet transfer mode became the one-pulse one-droplet transfer when the peak time was set to be between 1.5 and $3.0 \mathrm{~ms}$. It was also showed that a plasma and a metal vapor exhibited periodic behaviors in this transfer mode. In their study, SMAC (Simplified Marker And Cell) method [4] and VOF (Volume Of Fluid) method [5] were used to calculate the velocity and pressure fields of the arc plasma and to track the free surface of a molten metal, respectively. Hertel et al. calculated GMA welding phenomena on the doughnut-shape cathode and they also simulated behaviors of a plasma and molten metal droplets with changing of a current waveform [6]. Their simulation was conducted by one kind of finite volume methods and VOF method in three-dimensional computational domain with the rotationally periodic boundary [6] [7]. Their target was also pulse GMA welding and they calculated the temperature, the metal vapor concentration and the current density distributions at each point on the waveform. The droplet transfer mode obtained by this simulation showed good agreement with an experiment. They suggested that a molten metal droplet transfer was conducted by large axial velocity when the current density vector in radial direction became strong.

However to track the free surface of a molten metal smoothly, some high level techniques such as a VOF method [5] are required in traditional grid methods like above previous studies. For example, to apply the VOF method to the simulation, additional equations should be solved to determine the free surface. These 
equations make the computational costs increase. In this study, an SPH (Smoothed Particle Hydrodynamics) method that is suitable to simulate the dynamic behavior of fluid with large deformation is focused on, which can track the free surface easily. Then, the numerical model is developed on the basis of the SPH method to simulate droplet detachment in the projected transfer mode. The numerical simulation clarifies the transfer mechanisms. As a plasma heat source, the computational result obtained by the previous study [8] is used.

\section{Governing Equations}

\subsection{Density Homogenizing Algorithm}

In this study, the density homogenizing algorithm is used in order to apply a $\mathrm{SPH}$ method [9] that is originally used for compressible flows to incompressible flows such as molten metal. This algorithm is a computational technique which calculates a velocity field with the density homogenizing process using an iterative solution method based on a predictor-corrector method. The details of that density homogenizing algorithm are referred to the previous study [10]. That outline is described as follows. In the density homogenizing algorithm, at first, all fluid particles are moved according to their velocity predictors which are calculated by external forces and viscosity force obtained at the last time step. At this moment, the density field of the computational domain temporarily has a non-uniform distribution. To homogenize the density field, the predictors are corrected and all fluid particles are moved again according to these corrected predictors. This algorithm is a computational method to express the incompressible flow, in which the positions of particles are adjusted by these iterations until the density field in the whole region is homogenized.

\subsection{Smoothed Particle Hydrodynamics Method}

In the SPH method, physical quantities such as mass, energy, and so on are transported by fluid particles. A physical quantity $A$ at a certain position $a$ is written as

$$
A_{a}=\sum_{b} m_{b} \frac{A_{b}}{\rho_{b}} W_{a b},
$$

as the interactions with the adjacent particles $b$. Here, $a$ and $b$ are the indices of particles, $m$ is the mass, $\rho$ is the density, $W$ is the kernel function. In this study, M4-spline function is used as the kernel function, which is described as

$$
W_{a b}=\frac{1}{\pi h^{3}}\left\{\begin{array}{lr}
1.0-1.5\left(\frac{\left|\boldsymbol{I}_{a b}\right|}{h}\right)^{2}+0.75\left(\frac{\left|\boldsymbol{I}_{a b}\right|}{h}\right)^{3} & \left(0 \leq\left|\boldsymbol{I}_{a b}\right|<h\right) \\
2.0-3.0 \frac{\left|\boldsymbol{I}_{a b}\right|}{h}+1.5\left(\frac{\left|\boldsymbol{I}_{a b}\right|}{h}\right)^{2}-0.25\left(\frac{\left|\boldsymbol{I}_{a b}\right|}{h}\right)^{3} & \left(h \leq\left|\boldsymbol{I}_{a b}\right|<2 h\right) \\
0 & \left(2 h \leq\left|\boldsymbol{I}_{a b}\right|\right)
\end{array},\right.
$$

where $h$ is the kernel radius which is the same as the particle diameter and $l$ is 
the relative distance between particle $a$ and $b$. Using Equation (1), Equation (2) and the Navier-Stokes equation which expresses the motion of fluid, the acceleration of a particle is written as

$$
\frac{D \boldsymbol{u}_{a}}{D t}=-\sum_{b} m_{b}\left(\frac{p_{a}}{\rho_{a}{ }^{2}}+\frac{p_{b}}{\rho_{b}{ }^{2}}\right) \nabla_{a} W_{a b}+\frac{2 \delta}{\lambda_{a} n_{a} \rho_{a}} \sum_{b \neq a} \frac{\mu_{a}+\mu_{b}}{2}\left(\boldsymbol{u}_{b}-\boldsymbol{u}_{a}\right) W_{a b}+\frac{\boldsymbol{F}_{a}}{\rho_{a}},
$$

where $u$ is the velocity, $t$ is the time, $p$ is the pressure, $\operatorname{dim}$ is the dimension number, $\lambda$ is the parameter of moving particle semi-implicit method [11], $n$ is the number density, $\mu$ is the viscosity and $F$ is the external force. $\nabla_{a} W_{a b}$ is first derivative of the kernel function, which is expressed as

$$
\nabla_{a} W_{a b}=\frac{10}{7 \pi h^{4}}\left\{\begin{array}{lc}
-3.0 \frac{\left|\boldsymbol{I}_{a b}\right|}{h}+2.25\left(\frac{\left|\boldsymbol{I}_{a b}\right|}{h}\right)^{2} & \left(0 \leq\left|\boldsymbol{I}_{a b}\right|<h\right) \\
-3.0+3.0 \frac{\left|\boldsymbol{I}_{a b}\right|}{h}-0.725\left(\frac{\left|\boldsymbol{I}_{a b}\right|}{h}\right)^{2} & \left(h \leq\left|\boldsymbol{I}_{a b}\right|<2 h\right) \\
0 & \left(2 h \leq\left|\boldsymbol{I}_{a b}\right|\right)
\end{array} .\right.
$$

\subsection{Calculation of the Drag Force by the Shielding Gas}

Figure 1 shows the schematic illustration of wire, droplet and external forces acting on the droplet. As driving forces, the gravity force, the surface tension force, the pressure and the shearing force by shielding gas and the electromagnetic force caused by the welding current act on a wire and a molten metal droplet during GMA welding [12]. However, it is difficult to consider the pressure and shearing force separately using the velocity fields obtained by steady calculation [8] because with/without and direction of those forces are changed by the droplet positions and its shape. So in this study, the drag force including the effect of the pressure and shearing force by shielding gas is given to a droplet.

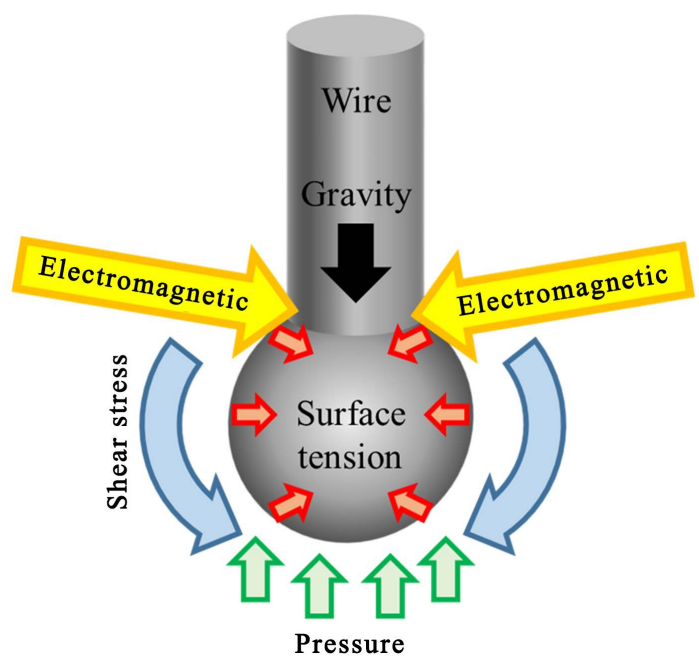

Figure 1. Schematic illustration of driving forces effecting on the droplet and the wire during a GMA welding. 
When a droplet is regarded as a perfect hard sphere, the drag force $F^{D}$ acting on the sphere is expressed as,

$$
F^{D}=\frac{1}{2 V} \bar{\rho} \bar{u}^{2} C_{D} \frac{\pi}{4} d^{2} .
$$

Here, $V$ is the volume of a particle, $\bar{\rho}$ and $\bar{u}$ are averaged density and averaged relative velocity of the shielding gas around a droplet. $d$ is the diameter of a droplet, which is set to be $1.5 \mathrm{~mm}$ as an imaginary droplet diameter. $C_{D}$ is the coefficient of the drag force [13] which is shown by,

$$
C_{D}=\left\{\begin{array}{lc}
\frac{24}{R e_{D}} & \left(R e_{D}<0.1\right) \\
\frac{24}{R e_{D}}\left(1+\frac{3}{16} R e_{D}-\frac{19}{1280} R e_{D}^{2}\right) & \left(0.1 \leq R e_{D}<1\right) \\
\frac{20.4}{R e_{D}}\left(1+0.136 R e_{D}^{0.803}\right) & \left(1 \leq R e_{D}<20\right) \\
\frac{R e_{D}}{20.4}\left(1+0.138 R e_{D}^{0.793}\right) & \left(20 \leq R e_{D}<100\right) \\
\frac{24}{R e_{D}}\left(1+0.125 R e_{D}^{0.72}\right) & \left(100 \leq R e_{D}<1000\right) \\
0.44 & \left(1000 \leq R e_{D}\right)
\end{array}\right.
$$

$R e_{D}$ is the Reynolds number and it can be described as,

$$
R e_{D}=\frac{\bar{\rho} \bar{u} d}{\bar{\mu}} .
$$

Where $\bar{\mu}$ is the averaged viscosity coefficient of a shielding gas around a molten metal droplet. The drag force in this model is calculated considering the effects of the shielding gas not only near the droplet but also around it. Therefore, the velocity, the temperature, the iron vapor concentration, the viscosity coefficient and the density of the shielding gas which are used to calculate Equation (5), Equation (6) and Reynolds number respectively should be obtained from not much on the grid point, but averaged value within some area. In this study, averaged velocity, averaged density and averaged viscosity coefficient at each $Z$ coordinate on $r=0 \mathrm{~m}$ are obtained using grid points within an area (from $r=0$ $\mathrm{m}$ to $2.25 \times 10^{-3} \mathrm{~m}$ in radial direction, $z=z \pm 2.25 \times 10^{-3} \mathrm{~m}$ in axial direction) in the result of previous study [8] shown in Figure 2. In this figure, dark gray regions show wire and base metal, respectively. Moreover, $r=0 \mathrm{~m}$ shows symmetrical axis. This previous study did not consider the motion of droplets for simplification. Actually, there are large variations of temperature between the surface of a moving droplet and plasma. Plasma properties such as density and viscosity are changed largely depending on temperature. In this study, to calculate the approximated drag force which acts on a droplet averagely, the averaged values of those properties are used. So, the averaged values are calculated in the area three times as large as the imaginary droplet diameter. Then, the drag force acting on a molten metal droplet is calculated by those averaged parameters. 


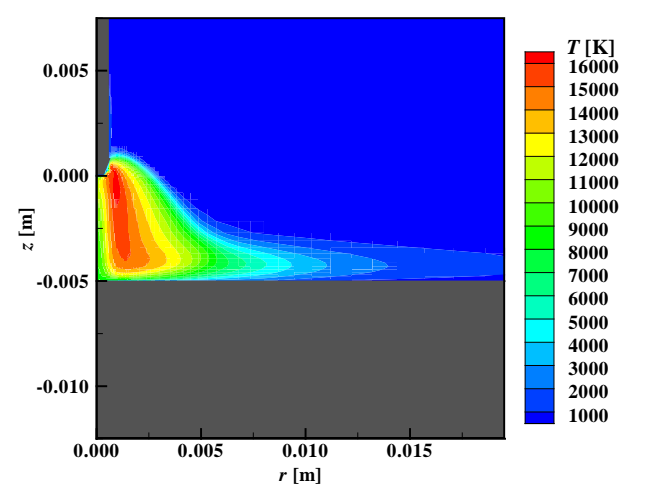

(a)

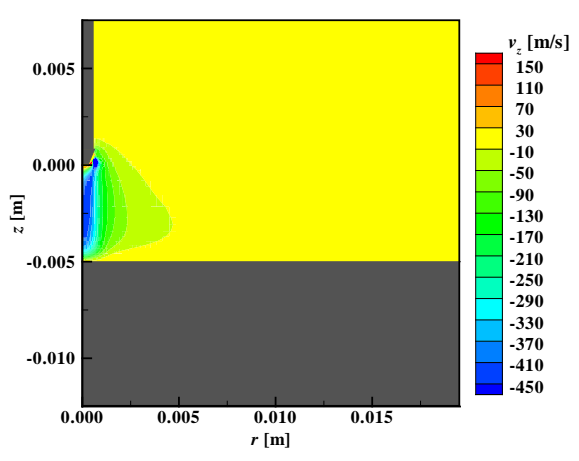

(b)

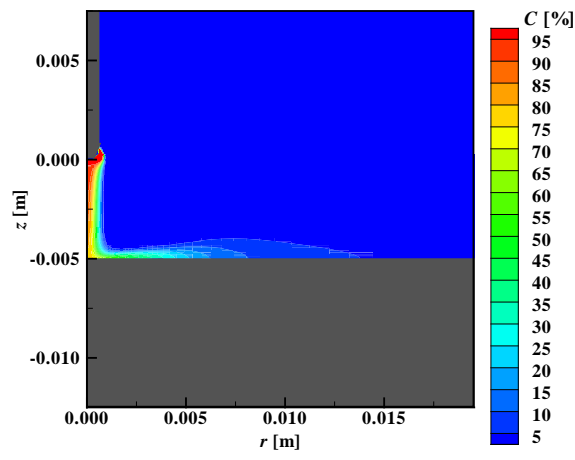

(c)

Figure 2. Calculation results using axisymmetric GMA model [8]. (a) Temperature distribution; (b) Velocity distribution in $z$ direction; (c) Iron vapor concentration distribution.

Figure 3 shows averaged distributions. In Figure 3, $z=0 \mathrm{~m}$ on horizontal axis shows the tip of a wire and $z=-5 \times 10^{-3} \mathrm{~m}$ means the base metal surface. Figure 4 shows the relationship between the temperature and the density or the viscosity coefficient of Ar gas for each mixing rate. These material properties are provided by Anthony B. Murphy [14]. Using these results and Equation (5), the drag force is calculated. Figure 5 shows the drag force distribution. The force becomes the strongest near the tip of a wire. This is because the drag force is proportional to square of the velocity. Strong drag force acts on the tip of a wire at which the velocity of a plasma flow is the largest. Since the force which is equivalent to $15 \mathrm{G}$ acts on a droplet at this point, it can be estimated that a droplet is accelerated largely by the shielding gas. The drag force at $z$ coordinate of each particle is obtained from Figure 5. Then, the accelerations which is given to particles are calculated using the force and the mass of an imaginary droplet.

\subsection{Driving Forces Acting on a Molten Metal Droplet}

Using the drag force obtained by Figure 5, the external force term of Equation (3) which is determine the velocity of particle $a$ is given as

$$
\boldsymbol{F}_{a}=\boldsymbol{j} \times \boldsymbol{B}+\boldsymbol{F}_{a}^{L}+\boldsymbol{F}_{a}^{L S}+\boldsymbol{F}_{a}^{D}+\rho_{a} \boldsymbol{g} .
$$

$\boldsymbol{j}, \boldsymbol{B}$ show the current density vector and the magnetic flux vector at each particle 


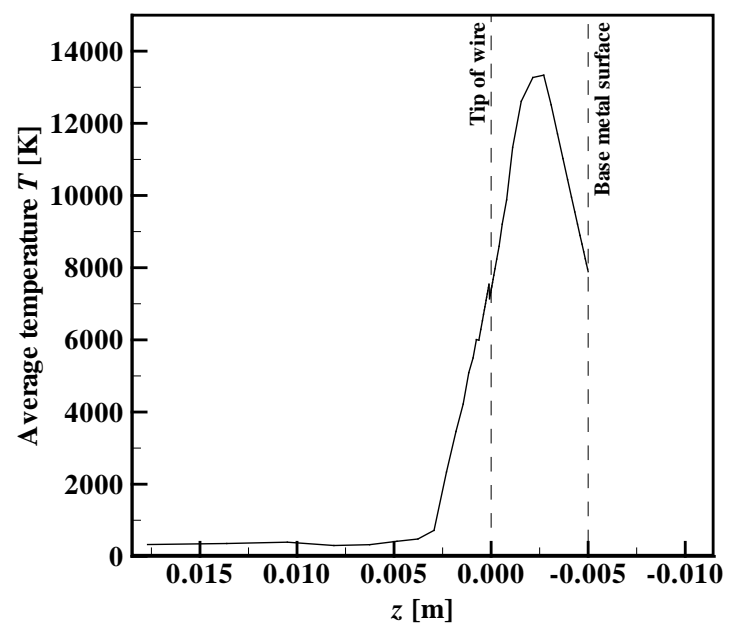

(a)

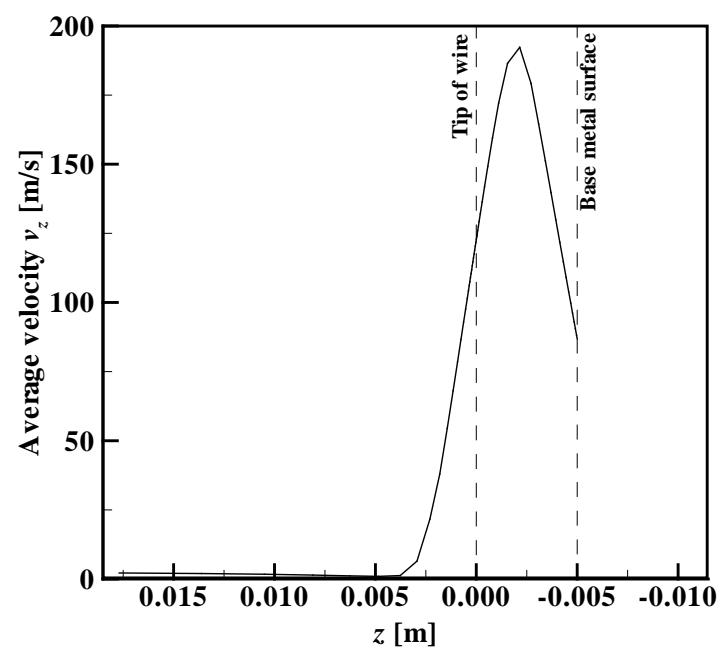

(b)

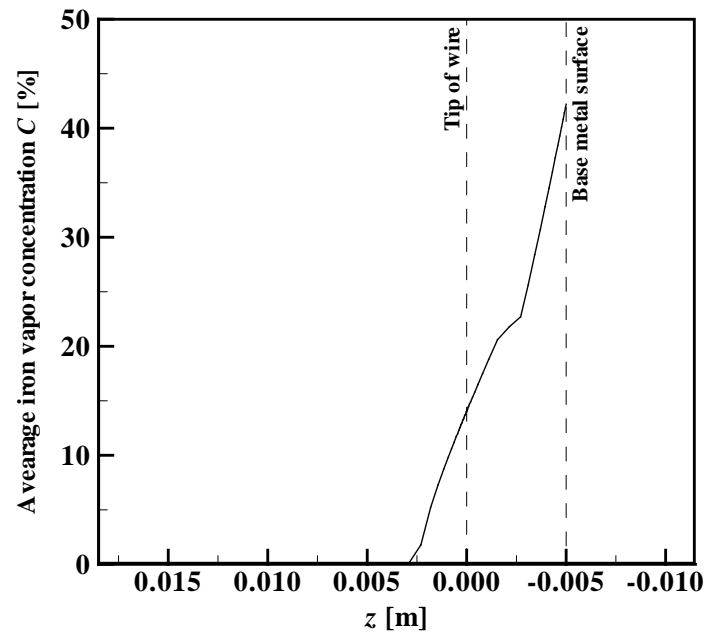

(c)

Figure 3. Averaged distributions at the axis in a GMA model. (a) Velocity distribution in $z$ direction; (b) Temperature distribution; (c) Iron vapor concentration distribution. 


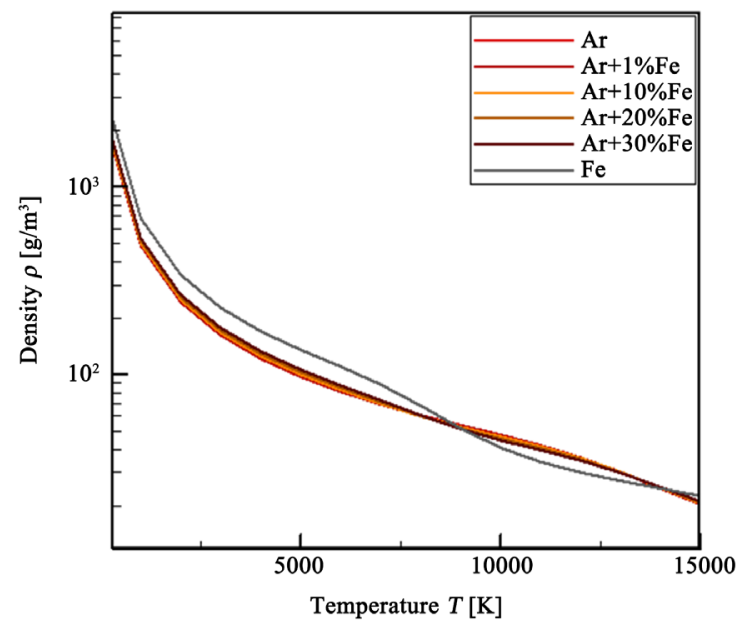

(a)

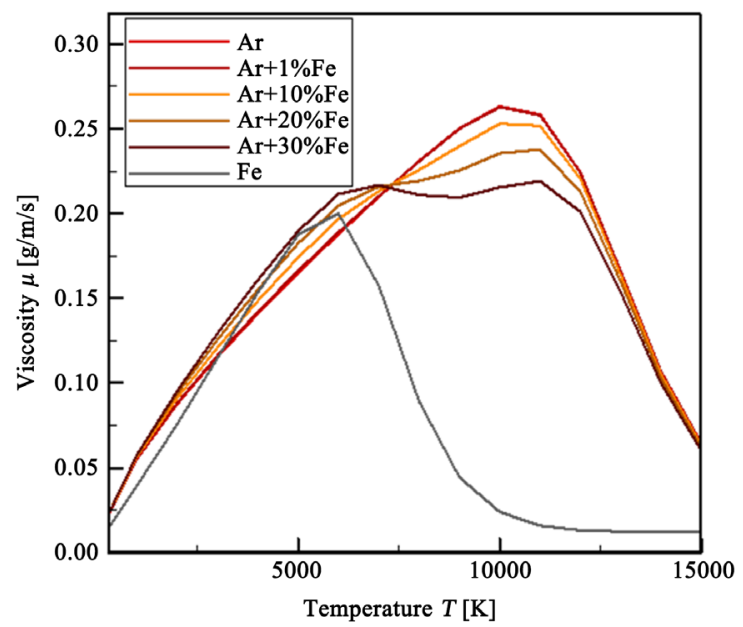

(b)

Figure 4. The relationship between temperature and density or viscosity of argon gas for each mixing rate. (a) Density; (b) Viscosity.

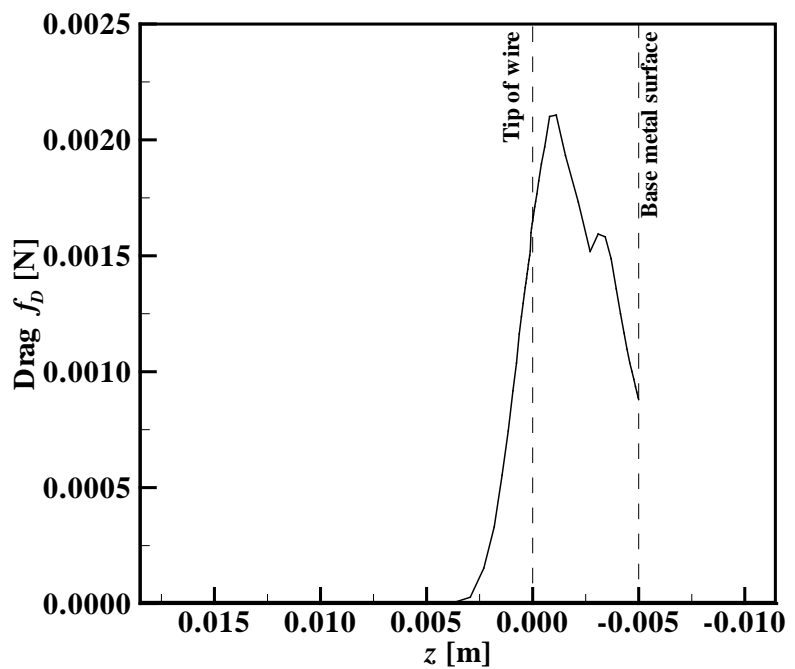

Figure 5. Drag force distribution around a wire. 
position and this term expresses the electromagnetic force acting on particles. In this calculation, the electromagnetic force distribution shown in Figure 6 obtained from the previous study [8] is given to the computational domain. The force at each particle position is calculated using the bilinear interpolation. $r$ is the horizontal axis of Figure 6 and it shows distance between the center of a wire and each particle on $x y$ plane. $z$ shows the distance from the solid-liquid interface of a wire to each particle positions. Moreover, a white line in the figure shows the boundary between a wire and a shielding gas. In the heat source model [8], it is difficult to determine the accurate solid-liquid interface because the wire shape is not changed. So the wire within $1.5 \mathrm{~mm}$ from the tip of it in Figure 6 is regarded as a molten metal and the solid-liquid interface of this simulation is set to be $1.5 \mathrm{~mm}$ from the wire tip. $\rho$ is the density, $\boldsymbol{F}^{L}, \boldsymbol{F}^{L S}$ are surface tension vectors obtained by the attractive force model, and $\boldsymbol{g}$ is the acceleration of gravity. Following the previous study [15], the surface tension vectors in the second term of right hand-side of Equation (8) is described as

$$
\begin{gathered}
\boldsymbol{F}_{a}^{L}=\left.\frac{1.2(1-\psi) \gamma h}{V} \sum_{b} f_{a b}^{\text {attract }}\right|_{a, b \in \text { Liquid }}, \\
\boldsymbol{F}_{a}^{L S}=\left.\frac{1.2 \psi \gamma h}{V} \sum_{b} f_{a b}^{\text {attract }}\right|_{a, b \in(\text { LiquidUSolid })} .
\end{gathered}
$$

$\psi$ is the constant to decide a contact angle. In this study, it is used as the parameter to express the relationship between solid and liquid, which is set to be 0.8 . $\gamma$ is the surface tension coefficient that is $1.0 \mathrm{~N} / \mathrm{m} . f_{a b}^{\text {attract }}$ is the weighted function which is determined by the distance between particle $a$ and $b$ [15]. These indices $L, S$ of surface tension vectors $\boldsymbol{F}^{L}$ and $\boldsymbol{F}^{L S}$ show the states of a particle. When both of particles $a$ and $b$ are liquid, both of Equation (9) and Equation (10) are calculated. On the other hand, when particle $a$ or $b$ is solid, only Equation (10) is calculated.

\section{Computational Conditions and Computational Domain}

Table 1 shows the computational conditions of this study. Material properties of the mild steel are given to all particles. Figure 7 shows the computational domain. A wire and a pool which is set bottom of the domain are composed of particles with $0.1 \mathrm{~mm}$ diameter. The solid particles of which a wire consists move with wire feed rate in $z$ direction. The tip of a wire at start of the simulation is set to be $11.8 \mathrm{~mm}$ height from bottom of the domain in $z$ direction.

In this study, the temperature change is not considered for simplification based on the assumption in which height of the solid-liquid interface is kept constant. This assumption means that the wire feed rate balances with the wire melting speed [12]. In actual gas metal arc welding, height of the solid-liquid interface is changed by the wire melting rate and wire feed rate. The wire melting speed is determined by mainly Joule heating and heat transfer with arc plasma. Increasing this melting speed makes height of the solid-liquid interface higher. 


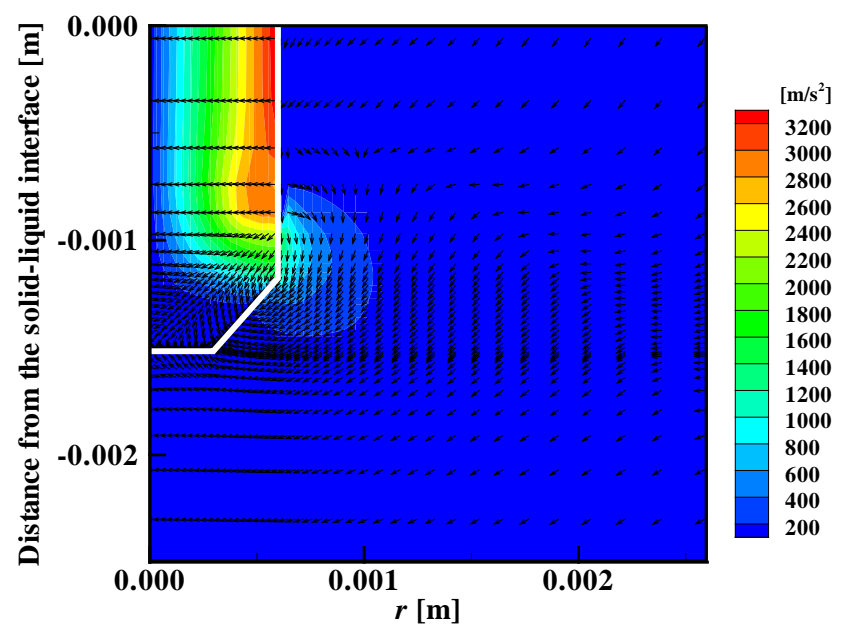

Figure 6. The Lorentz force distribution in a GMA welding [8].

Table 1. Computational conditions.

\begin{tabular}{cc}
\hline Diameter of particle & $\phi 0.1 \mathrm{~mm}$ \\
Density of particle & $7850 \mathrm{~kg} / \mathrm{m}^{3}$ \\
Time step & $1.0 \times 10^{-2} \mathrm{~ms}$ \\
Acceleration of gravity & $-9.8 \mathrm{~m} / \mathrm{s}^{2}$ \\
Surface tension coefficient & $1.0 \mathrm{~N} / \mathrm{m}$ \\
Viscosity coefficient & $4.0 \times 10^{-3} \mathrm{~Pa} \cdot \mathrm{s}$ \\
Wire feed rate & $7.9 \mathrm{~m} / \mathrm{min}$ \\
\hline
\end{tabular}

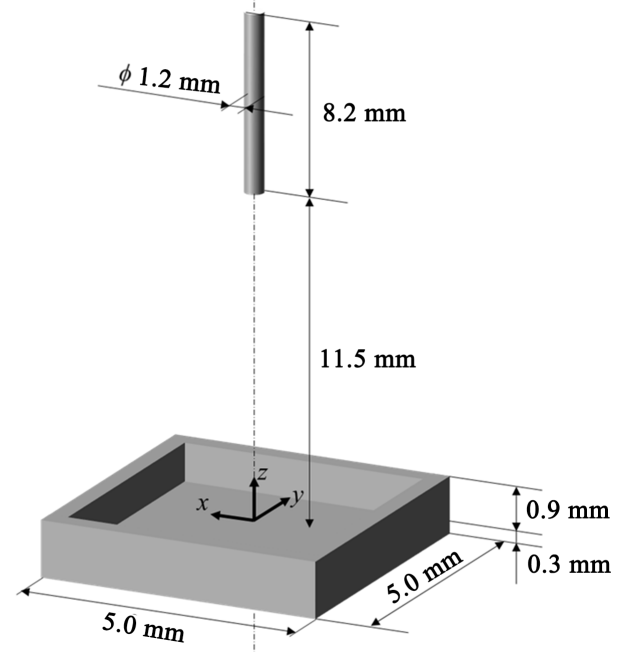

Figure 7. Computational domain.

On the other hand, height of the solid-liquid interface becomes lower by increase wire feed rate which is the velocity to feed the wire. To express this wire melting phenomena in this simulation, solid particles which make the wire become liquid state instantaneously when they move to the position that is lower than the solid-liquid interface. 


\section{Results and Discussion}

Figure 8 shows computational results at each time. The size of a molten metal droplet becomes large the same as the wire diameter. Then, it is detached near the top of the droplet. The droplet detachments regularly occur about every 10 ms. In this simulation, the droplet transfer frequency is determined $100 \mathrm{~Hz}$ because 10 droplets are formed for $0.1 \mathrm{~s}$ through the droplet growth and detachment processes. The droplet transfer mode obtained from this calculation is regarded as a projected transfer mode, in which the liquid column grows about 1 $\mathrm{mm}$ and a droplet grows up until its diameter becomes the same as a wire diameter, after that it is detached from the tip of the column. Figure 9 shows velocity fields from start of the droplet formation to end of the detachment of it at a wire tip. These figures show $y z$ cross-sections at center of a wire in which particles within $0 \pm 0.1 \mathrm{~mm}$ in $x$ direction are drawn. Each point shows center of a particle and its color indicates the velocity in $z$ direction. Arrow heads express velocity of particles. Figure 9(a) shows the instantaneous image in which a molten metal droplet is just detached from the tip of a wire. When the tip of an extended liquid column is broken away and a droplet is formed, large surface tension force acts on the wire tip because the liquid column attaching to the tip becomes thin. Velocity vectors of liquid particles in the column reverse upward gradually from the tip of a wire by the surface tension force and the liquid column moves upward (Figure 9(b)). On the other hand, the molten metal near the solid-liquid interface spreads to outside by the pressure from a wire tip (Figure 9(c)). The surface tension force near the tip of a wire becomes weaker by increasing of the curvature radius at the tip. After that, the downward force due to the electromagnetic force, the drag force and the gravity becomes dominant. Therefore, velocity vectors of liquid particles reverse downward (Figure 9(d)). Since molten metal is supplied by the wire feed, the liquid column grows again and its diameter becomes large the same as a wire diameter with the turn back of velocity fields (Figure 9(e)). Thereafter, the velocity of a liquid column becomes large with the droplet growth at the tip of the column and the column is pinched toward the center of a wire by the electromagnetic force. The flow from the solid-liquid interface to a molten metal droplet becomes faster than the supply of the molten metal by the wire feed (Figure $9(f)$ ). Thus, the bridge is made by the flow because the molten metal of which a liquid column consists flows to a molten metal droplet (Figure $9(\mathrm{~g})$ ), and grown droplet is detached from the tip of a wire (Figure 9(h)). In this simulation, it is clarified that the droplet transfer phenomena are conducted with those droplet growth and detachment processes. The averaged diameter and the averaged velocity in $z$ direction of droplets which fell to $5 \mathrm{~mm}$ from the detached position are estimated to be $1.26 \mathrm{~mm}$ and -1.56 $\mathrm{m} / \mathrm{s}$, respectively.

To compare with actual projected transfer mode, the GMA welding process with typical conditions (welding current was $313 \mathrm{~A}$, arc voltage was $36.8 \mathrm{~V}$ ) was carried out and molten metal droplet transfers were taken by high speed camera 

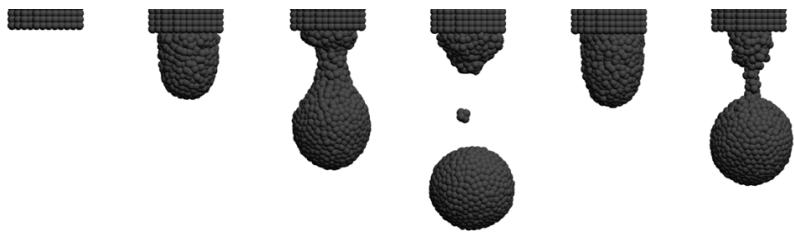

(a)



(h) (c)

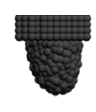

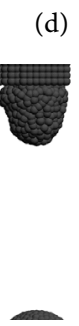

(j) (e)

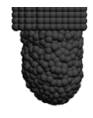

$(\mathrm{k})$ (f)

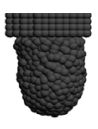

(1)

Figure 8. Time evolution of the molten metal droplet. (a) $t=0 \mathrm{~s}$; (b) $t=5 \mathrm{~ms}$; (c) $t=10 \mathrm{~ms}$; (d) $t=11 \mathrm{~ms}$; (e) $t=15 \mathrm{~ms}$; (f) $t=20$ ms; (g) $t=30 \mathrm{~ms}$; (h) $t=40 \mathrm{~ms}$; (i) $t=50 \mathrm{~ms}$; (j) $t=70 \mathrm{~ms}$; (k) $t=$ $90 \mathrm{~ms}$; (l) $t=100 \mathrm{~ms}$.

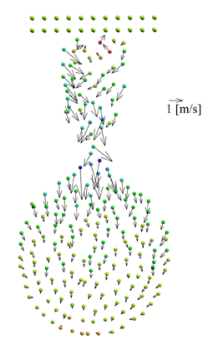

(a)

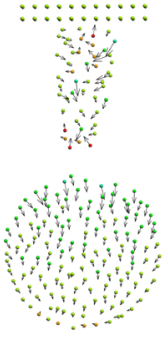

(b)
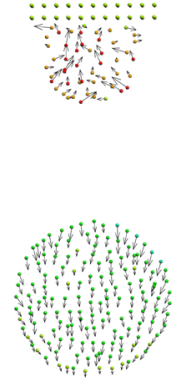

(c)

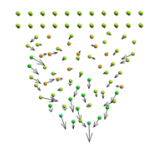

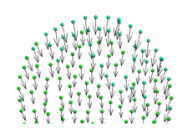

(d)

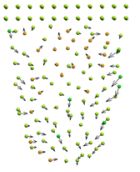

(e)

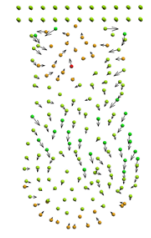

(f)

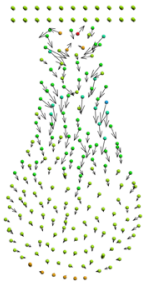

(g)

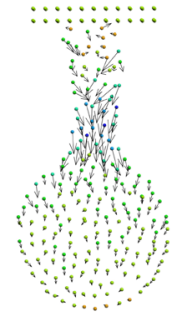

(h)

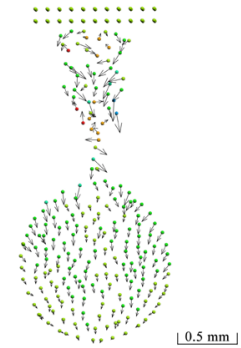

(i)

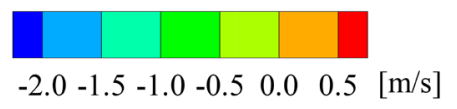

Figure 9. Time evolution of the molten metal droplet with velocity field (from $t=$ $11.00 \mathrm{~ms}$ ). (a) $t=11.00 \mathrm{~ms}$; (b) $t=11.21 \mathrm{~ms}$; (c) $t=11.84 \mathrm{~ms}$; (d) $t=12.54 \mathrm{~ms}$; (e) $t$ $=15.00 \mathrm{~ms}$; (f) $t=17.33 \mathrm{~ms}$; (g) $t=19.41 \mathrm{~ms}$; (h) $t=20.21 \mathrm{~ms}$; (i) $t=20.88 \mathrm{~ms}$.

(MIROeX). Frame rate and exposure time of the camera were 2000 fps and $2 \mu$ s, respectively. In this experiment, the mixture gas was used because the transfer mode in pure Ar atmosphere became from the streaming transfer to the globular transfer by reducing a welding current and the projected transfer mode was not 
obtained. Figure 10 shows the experimental results. In addition, Table 2 shows the comparison results between this experiment and simulation. As a result, the droplet transfer mode in a MAG welding became a projected transfer mode. So, it was made clear that numerical results showed a tendency the same as this experiment. Figure 10(a) and Figure 10(b) show the instantaneous images right before and after a molten metal droplet was detached. From computational results in previous studies, it can be estimated that tip of the solid-liquid interface is located in the arc plasma [6] [16]. Therefore, it was defined the length of liquid column in the experiment was from top of the column to bottom of it in the arc plasma. The measurement was conducted using Image J [17]. The length of a liquid column in Figure 10(b) was $1.15 \mathrm{~mm}$, which agreed with the computation $(1.13 \mathrm{~mm})$. After that, a molten metal droplet fell in an arc plasma to a weld pool (Figure 10(b) and Figure 10(h)) and next droplet was detached (Figure 10(i)). The velocity and the diameter of a droplet right before it reached a weld pool surface were about $-1.40 \mathrm{~m} / \mathrm{s}$ and $1.14 \mathrm{~mm}$, respectively (Figure 10(f), Figure 10(g)).

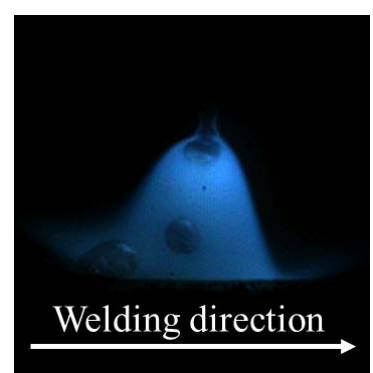

(a)

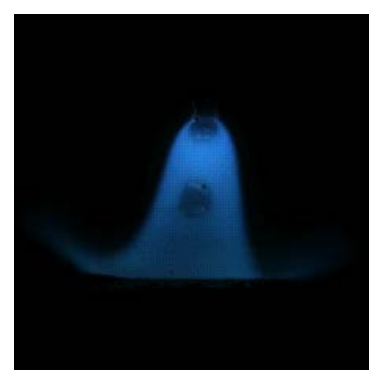

(d)

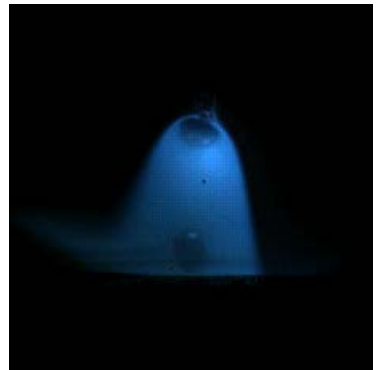

(g)

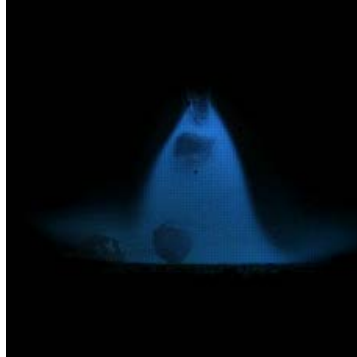

(b)

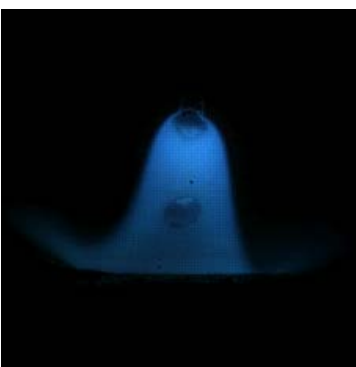

(e)

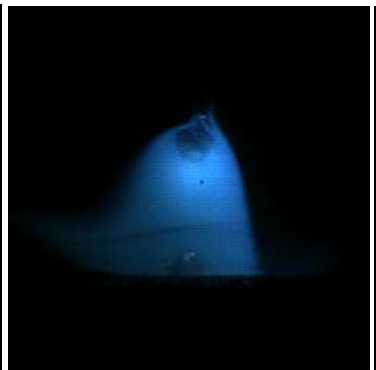

(h)

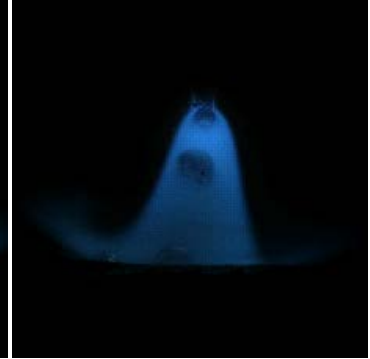

(c)

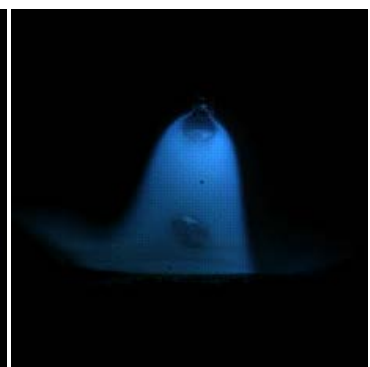

(f)

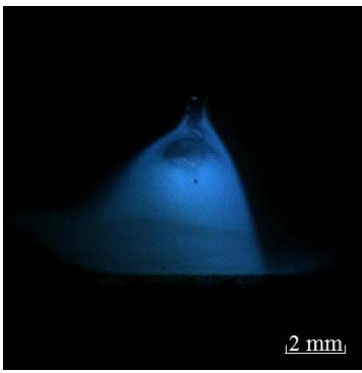

(i)

Figure 10. Experimental results using mixing gas $\left(80 \% \mathrm{Ar}+20 \% \mathrm{CO}_{2}\right)$. (a) $t=0 \mathrm{~s}$; (b) $t=0.5 \mathrm{~ms}$; (c) $t=1.0 \mathrm{~ms}$; (d) $t=1.5 \mathrm{~ms}$; (e) $t=2.0 \mathrm{~ms}$; (f) $t=2.5 \mathrm{~ms}$; (g) $t=3.0$ ms; (h) $t=3.5 \mathrm{~ms}$; (i) $t=4.0 \mathrm{~ms}$. 
Table 2. Comparison between experimental and simulation results.

\begin{tabular}{ccc}
\hline & Simulation & Experiment \\
\hline Diameter of droplet $[\mathrm{mm}]$ & 1.26 & 1.14 \\
Velocity of droplet $[\mathrm{m} / \mathrm{s}]$ & 1.56 & 1.40 \\
Length of fluid column $[\mathrm{mm}]$ & 1.13 & 1.15 \\
\hline
\end{tabular}

As stated above, the diameter of a wire, the length of a liquid column, the velocity of a droplet right before it reached a weld pool obtained by simulation showed good agreement with experiment.

\section{Conclusions}

In this study, three-dimensional computational model was developed by an incompressible SPH method in which it was easy to track the free surface and calculated motion of the fluid with large deformation stably. Then, the numerical simulation of the molten metal droplet transfer during a GMA welding was carried out. The conclusions of this study can be summarized as follows:

1) An incompressible SPH method simulated that 10 droplets were formed for $0.1 \mathrm{~s}$ through growth and detachment processes at the tip of a wire.

2) The droplet transfer mode obtained from this calculation was regarded as a projected transfer mode, in which the liquid column grew about $1 \mathrm{~mm}$ and a droplet grew up until its diameter became large the same as a wire diameter, after that it was detached from the tip of the column.

3) The diameter of a wire, the length of a liquid column, the velocity of a droplet right before it reached a weld pool obtained by simulation showed good agreement with experiment.

\section{References}

[1] Liu, S. and Siewert, T.A. (1989) Metal Transfer in Gas Metal Arc Welding: Droplet Rate. Welding Journal, 68, 52-58.

[2] Girish, J. and Chauhan, B.J. (2015) Effect of Shielding Gases on Austenitic Stainless Steel Overlay by FCAW Process on Low Alloy Steel. International Journal of Science and Research, 4, 987-993.

[3] Ogino, Y., Hirata, Y. and Asai, S. (2017) Numerical Simulation of Metal Transfer in Pulsed-MIG Welding. Welding in the World, 61, 1289-1296.

https://doi.org/10.1007/s40194-017-0492-3

[4] Amsden, A.A. and Harlow, F.H. (1970) The SMAC Method: A Numerical Technique for Calculating Incompressible Fluid Flows. Los Alamos Science Laboratory Report, LA-4370.

[5] Hirt, C.W. and Nichols, B.D. (1981) Volume of Fluid (VOF) Method for the Dynamics of Free Boundaries. Journal of Computational Physics, 39, 201-225. https://doi.org/10.1016/0021-9991(81)90145-5

[6] Hertel, M., Trautmann, M., Jäckel, S. and Füssel, U. (2017) The Role of Metal Vapour in Gas Metal Arc Welding and Methods of Combined Experimental and $\mathrm{Nu}$ merical Process Analysis. Plasma Chemistry and Plasma Processing, 37, 531-547. 
https://doi.org/10.1007/s11090-017-9790-1

[7] Hertel, M., Spille-Kohoff, A., Füssel, U. and Schnick, M. (2013) Numerical Simulation of Droplet Detachment in Pulsed Gas-Metal Arc Welding Including the Influence of Metal Vapour. Journal of Physics D: Applied Physics, 46, 224003. https://doi.org/10.1088/0022-3727/46/22/224003

[8] Tsujimura, Y. (2012) Study of Arc Phenomena with Metal Vapor and the Heat Source Characteristics during Gas Metal Arc Welding. Ph.D. Thesis, Osaka University, Osaka.

[9] Monaghan, J.J. (1994) Simulating Free Surface Flows with SPH. Journal of Computational Physics, 110, 399-406. https://doi.org/10.1006/jcph.1994.1034

[10] Shigeta, M., Watanabe, T., Izawa, S. and Fukunishi, Y. (2009) Incompressible SPH Simulation of Double-Diffusive Convection Phenomena. International Journal of Emerging Multidisciplinary Fluid Sciences, 1, 1-18. https://doi.org/10.1260/1756-8315.1.1.1

[11] Koshizuka, S. and Oka, Y. (1996) Moving-Particle Semi-Implicit Method for Fragmentation of Incompressible Fluid. Nuclear Science and Engineering, 123, 421-434. https://doi.org/10.13182/NSE96-A24205

[12] Ogino, Y. and Hirata, Y. (2015) Numerical Simulation of Metal Transfer in Argon Gas-Shielded GMAW. Welding in the World, 59, 465-473. https://doi.org/10.1007/s40194-015-0221-8

[13] Bhattacharyya, D. and Gauvin, W.H. (1975) Modeling of Heterogeneous Systems in a Plasma Jet Reactor. AIChE Journal, 21, 879-885. https://doi.org/10.1002/aic.690210508

[14] Murphy, A.B. (1997) Transport Coefficients of Helium and Argon-Helium Plasmas. IEEE Transactions on Plasma Science, 25, 809-814.

[15] Ito, M. (2015) Numerical Simulation of Flow Phenomena in Arc Welding Process by Incompressible SPH Method. Ph.D. Thesis, Tohoku University, Sendai.

[16] Ogino, Y., Hirata, Y. and Murphy, A.B. (2016) Numerical Simulation of GMAW Process Using Ar and an Ar- $\mathrm{CO}_{2}$ Gas Mixture. Welding in the World, 60, 345-353. https://doi.org/10.1007/s40194-015-0287-3

[17] Schneider, C.A., Rasband, W.S. and Eliceiri, K.W. (2012) NIH Image to ImageJ: 25 Years of Image Analysis. Nature Methods, 9, 671-675.

https://doi.org/10.1038/nmeth.2089 


\section{Nomenclature}

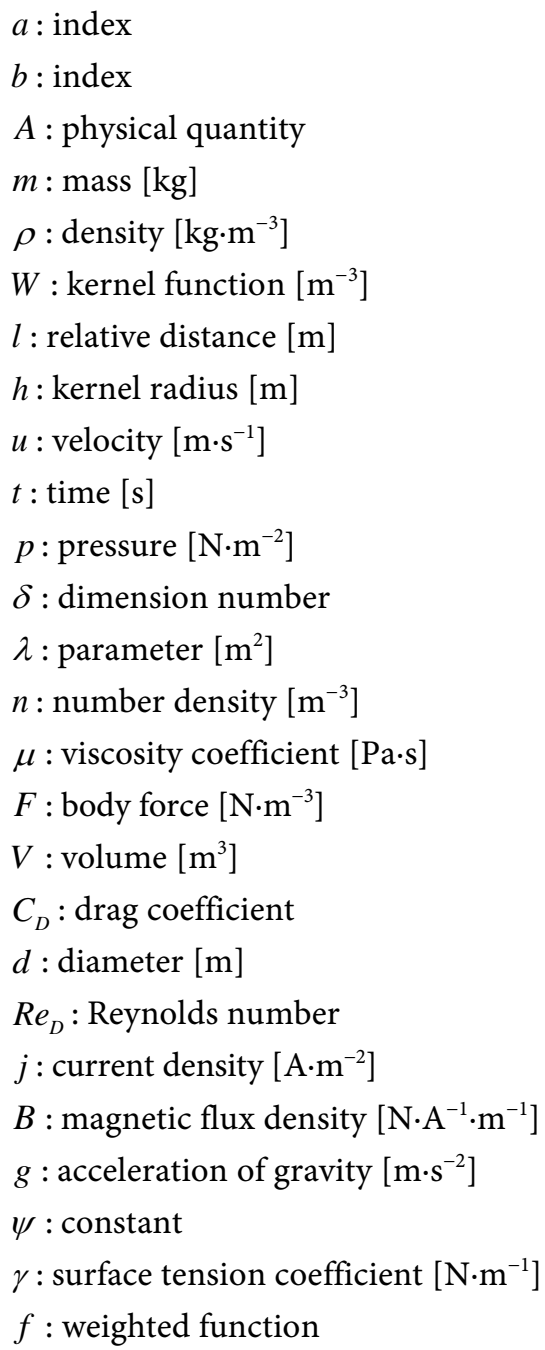

